\title{
Crustaceans (Decapoda \& Stomatopoda) from Golfo Dulce (Pacific, Costa Rica) in the collection of the Museum of Zoology, University of Costa Rica
}

\author{
Rita Vargas-Castillo ${ }^{1,2}$ iD \& José A. Vargas-Zamora ${ }^{1,3}$ \\ 1. Universidad de Costa Rica, Escuela de Biología, 11501-2060, San José, Costa Rica \\ 2. Universidad de Costa Rica, Escuela de Biología, Museo de Zoología, 11501-2060, San José, Costa Rica; \\ rita.vargas@ucr.ac.cr \\ 3. Universidad de Costa Rica, Centro de Investigación en Ciencias del Mar y Limnología (CIMAR), 11501-2060, San José, \\ Costa Rica; jose.vargas@ucr.ac.cr
}

Recibido 14-I-2020 • Corregido 15-IV-2020 • Aceptado 28-IV-2020

DOI: https://doi.org/10.22458/ urj.v12i1.2736

\begin{abstract}
Introduction: The availability of recently updated lists of species from a particular area is an important first step to evaluate changes in species composition and abundance. Golfo Dulce is a fjord-like embayment with relatively pristine shores and relatively deep waters $(200 \mathrm{~m})$ that have been sampled occasionally for crustaceans. Methods: In this study the all species from Golfo Dulce deposited in the collection of the University of Costa Rica Museum of Zoology were listed and scientific names were updated. The MZUCR catalog number, station, substrate type, and year or collection were included for each species. Results: A total of 106 species were listed. Neogonodactylus zacae was present in four of the 19 stations represented in the collections for Golfo Dulce. Nine species were present in three stations, 19 in two stations and the remaining 77 in one station each. The families with most species were Ocypodidae y Palaemonidae (7) and Porcellanidae (14). Conclusions: Is necessary to improve our knowledge of the decapods and stomatopods fauna from Golfo Dulce through further samplings. Intertidal sites such as Golfito and Rincón bays have been poorly sampled as well as intertidal and subtidal environment from the western and eastern shores. However, the fauna is relatively rich when it is related to the area of Golfo Dulce $\left(750 \mathrm{~km}^{2}\right)$ and is indicative of a relatively undisturbed environment. Updated management policies are urgently needed and this list of species is a step towards this goal.
\end{abstract}

Keywords: Biodiversity, eastern Tropical Pacific, Stomatopoda, Dendrobranchiata, Caridea, Anomura, Brachyura.
RESUMEN. "Crustáceos (Decapoda \& Stomatopoda) del Golfo Dulce (Pacífico, Costa Rica) en la colección del Museo de Zoología, Universidad de Costa Rica". Introducción: Las listas actualizadas de especies son un primer paso para evaluar cambios temporales en composición y abundancia. Golfo Dulce es un cuerpo de agua marino semejante a un fiordo, con costas poco alteradas y aguas relativamente profundas $(200 \mathrm{~m})$. Métodos: Elaboramos una lista taxonómicamente actualizada de las especies de macro-crustáceos recolectados en Golfo Dulce y depositadas en la colección del Museo de Zoología de la Universidad de Costa Rica (MZUCR). El número de catálogo del MZUCR, estación, tipo de sustrato, y año de colecta fueron anotados para cada especie. Resultados: Hay 106 especies en la lista. Neogonodactylus zacae estuvo en cuatro de las 19 estaciones representadas en las colecciones para Golfo Dulce. Nueve especies en tres estaciones, 19 en dos estaciones y las restantes 77 en una estación cada una. Las familias con más especies fueron Ocypodidae y Palaemonidae (7) y Porcellanidae (14). Conclusiones: Se requieren más muestreos, particularmente en sitios entre mareas como las bahías de Golfito y Rincón; y fondos de entre mareas y submareales de las costas oeste y este. La fauna es relativamente rica considerando el área de Golfo Dulce $\left(750 \mathrm{~km}^{2}\right)$ y es indicadora de un ambiente relativamente inalterado. Se requiere urgentemente de políticas de manejo actualizadas y esta lista en un paso hacia ese objetivo.

Palabras clave: Biodiversidad, Pacifico Tropical Este, Stomatopoda, Dendrobranchiata, Caridea, Anomura, Brachyura. 
Golfo Dulce is a deep embayment on the Pacific coast (08 $30^{\prime} \mathrm{N} \& 83^{\circ} 20^{\prime} \mathrm{W}$ ) of Costa Rica. The water dynamics of the system were described by Svendsen, Rosland, Myking, Vargas, Lizano and Alfaro (2006). The gulf is characterized by an inner basin $(200 \mathrm{~m})$ and a sill $(70 \mathrm{~m})$ at the entrance (Fig. 1). The bathymetry of the gulf was described by Hebbeln, Beese and Cortés (1996). The presence of a deep basin, steep shores and a sill results in Golfo Dulce being considered essentially a tropical fjordlike embayment (Wolff, Hartmann, \& Koch, 1996). Narrow shores with fringing mangrove forests, rocky outcrops, decaying coral reefs, and sandy beaches surround most of Golfo Dulce (Cortés, 1990; Samper-Villarreal \& Silva-Benavides, 2015). Hypoxic and anoxic conditions are found in waters deeper than 100m (Córdoba \& Vargas, 1996; Dalsgaard, Canfield, Peterson, Thamdrup, \& AcuñaGonzález, 2003), and no macrofaunal organisms have been found living in the anoxic basin sediments (León-Morales \& Vargas, 1998).

Information about the macro-crustacean fauna of Golfo Dulce is scarce. The most important survey was conducted by the R.V. Victor Hensen (1993-1994) which collected crustaceans over a depth range of 15 to $200 \mathrm{~m}$ using Otter and beam trawls. A preliminary list of 50 macro-crustaceans from Golfo Dulce collected during the R.V. Victor Hensen survey was published by Vargas, Jesse and Castro (1996). In addition, Castro and Vargas (1996) listed 68 species of macro-crustaceans reported in the literature for Golfo Dulce and included additional data for those collected during the survey. The spatial distributions of the species collected by the research vessel were analysed by Jesse (1996). Voucher specimens from most of the survey were deposited in the collection of the Museum of Zoology, University of Costa Rica (MZUCR). Other specimens deposited in the MZUCR were obtained over the years during occasional visits to different sites around the estuary, but a planned inventory of the crustacean fauna of the estuary is yet to be conducted.

Golfo Dulce is under the increasing pressure of local, regional and global stressors, such as pollution, coastal development, extraction of resources, and climate change (Morales-Ramírez, 2011, Morales-Ramírez, Acuña-González, Lizano, Alfaro, \& Gómez, 2015). A trophic model in Golfo Dulce considering the key roles of the crustaceans and other groups was developed by Woff et al. (1996), but it needs to be updated. In this context, information on the biodiversity of Golfo Dulce is urgently needed as input in order to improve the trophic model and to develop of new management policies at the ecosystem level. Data on the previously recorded species from the gulf is important to evaluate changes in biodiversity over time and to identify sites of relevance for future surveys. Thus, the objective of this report is to provide an updated list of species of macro-crustaceans (Decapoda and Stomatopoda) from Golfo Dulce deposited in the collection of the UCR-Museum of Zoology, identify gaps of information, and make suggestions for a future survey of the fauna.

\section{MATERIALS AND METHODS}

The crustacean collection of the Museum of Zoology of the University of Costa Rica (MZUCR) is composed with three components. First, the collection of specimens preserved in $70 \%$ ethanol in labeled glass jars. Second, a dossier of handwritten catalog cards, with data for each collecting site. Third, a digital file filled with data obtained from the catalog cards. All catalog entries of marine macrocrustacean species belonging to Golfo Dulce were selected from the digital file. The handwritten cards and the specimen collection were reviewed when sampling data was not clear and/or the species identification was necessary to double-check records. A list of species was assembled, arranged in alphabetical order, and a code number assigned to each one. The list includes the presently accepted species name, authority, catalog number, station and year of collection. Other information such as depth (intertidal, subtidal) and substrate description (rocks, coral, sediment, other) was also included, when available. Two Golfo Dulce species are listed in the cards with no other station data. Most of the 
specimens in the collection were collected by hand and occasionally by SCUBA while those from the R.V. Victor Hensen expedition were captured with otter and beam trawl nets. All species names were verified for their currently accepted name based in the web page World Register of Marine Species (WORMS).

Ethical, conflict of interest and financial statements: The authors have fully complied with all pertinent and legal requirements both during the study and in the production of the manuscript. We state that there are no conflicts of interest of any kind. The financial sources are fully and clearly stated in the acknowledgements section and we fully.

\section{RESULTS}

In total, there were 19 stations for which information was available (Fig. 1). Intertidal and SCUBA diving stations were assigned a code number, while those from trawl nets were identified with capital letters. A total of 106 species of macrocrustaceans with 173 entries was available for Golfo Dulce and catalogued in the collection of the Museum of Zoology (Appendix 1). Of this total, 102 are decapods and four are stomatopods. The earliest collection records are from 1969, followed by a time gap, until 1990 and 1993-1995 when 83 (48\%) new entries were listed, many as the result of the R.V. Victor Hensen survey. The next intensive sampling effort took place during 2012-2013. Thirty-nine of the collecting sites were coral rocks, while 32 stations were from sediments. Most of the collecting sites were intertidal. The maximum depth sampled was $200 \mathrm{~m}$ (Appendix 1).

The 102 species of decapods were distributed into 32 families, while the four species of stomatopods were represented by two (Table 1). The most specious family was the Porcellanidae, with 14 species. Other families, represented by five or more species, are: Ocypodidae (7), Palaemonidae (7), Alpheidae (6), Diogenidae (6), Xanthidae (6), Mithracidae (5), and Portunidae (5). Eleven families were represented by only one species (Table 1 ). There were ten most frequently species collected in the 19 stations (Table 2). Only one species, the stomatopod Neogonodactylus zacae, was collected at four stations. Nine species were found in three stations, 19 in two stations and the remaining 77 in only one station each. Station 12 had the most species (21), followed by stations 7 (17), 10 (16), 9 (15), and 3 (14). The other stations had less than 10 species each. Stations 1, 13, had one species each (Table 2). The R.V. Victor Hensen stations located near the sill and at the mouth of Golfo Dulce $(F, H$, and $G, I)$ included 10, 5, 6, and 6 species, respectively. Those stations in deeper waters inside the gulf had 1 (L) and $3(\mathrm{M})$ species. 


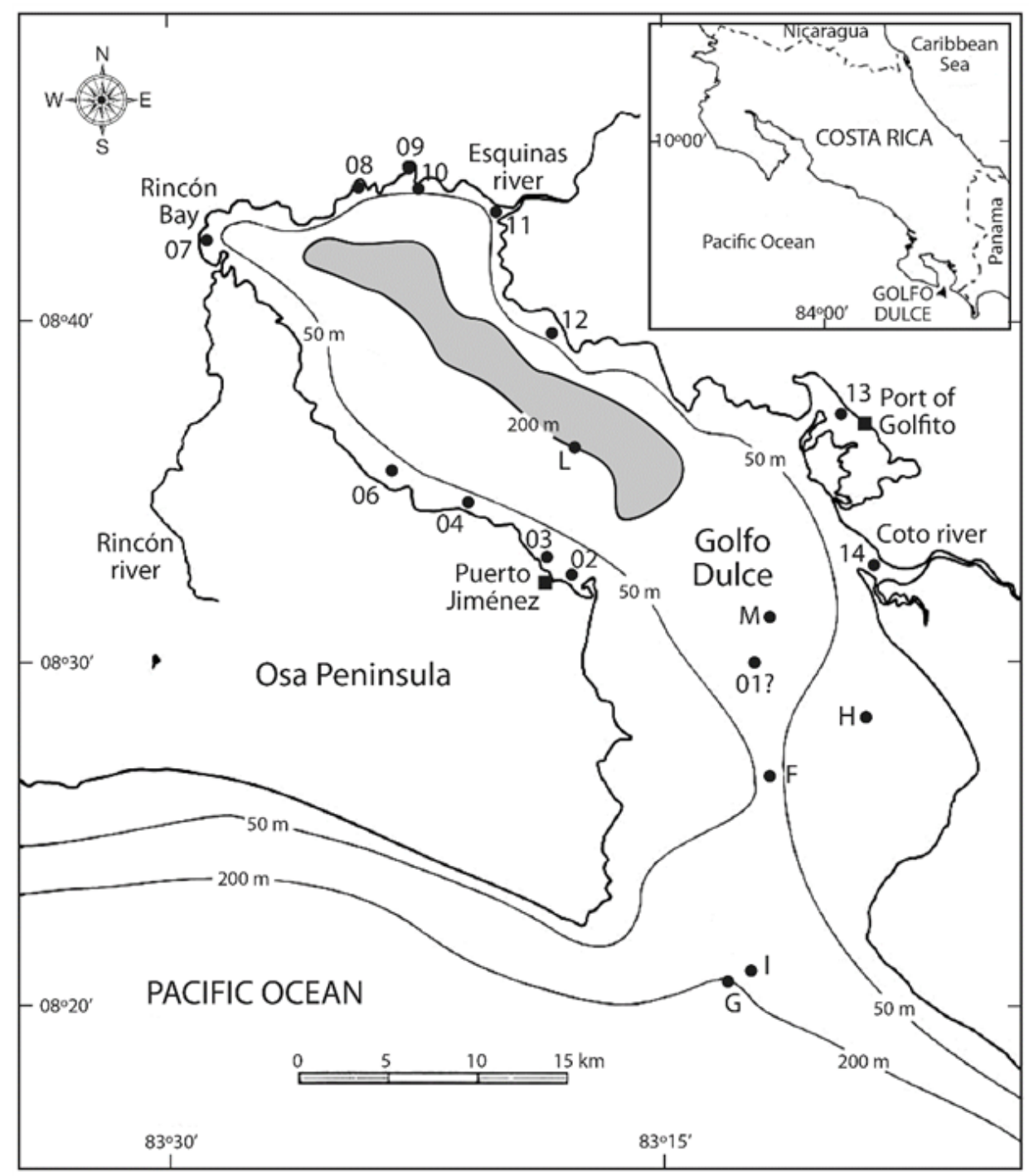

Fig. 1. Location of the stations reported in this study in Golfo Dulce. 2-14, intertidal and shallow water; F, G, H, I, M, deep-water stations from the R.V. Victor Hensen survey (1993 / 1994). The gray shaded area encloses basin depths of 200 to $215 \mathrm{~m}$. Station F is located at the sill $(70 \mathrm{~m})$. 


\section{TABLE 1}

Families of crustaceans and number of species for each family catalogued in the collection of the Museum of Zoology. Golfo Dulce, Pacific coast of Costa Rica

\begin{tabular}{|c|c|}
\hline DECAPODA & No. species \\
\hline Alpheidae & 6 \\
\hline Calappidae & 1 \\
\hline Callianideidae & 1 \\
\hline Cancridae & 1 \\
\hline Chasmocarcinidae & 1 \\
\hline Diogenidae & 6 \\
\hline Dynomenidae & 1 \\
\hline Gecarcinidae & 1 \\
\hline Grapsidae & 2 \\
\hline Hippidae & 1 \\
\hline Hippolytidae & 2 \\
\hline Leucosiidae & 3 \\
\hline Mithracidae & 5 \\
\hline Munididae & 2 \\
\hline Ocypodidae & 7 \\
\hline Oziidae & 3 \\
\hline Paguridae & 3 \\
\hline Palaemonidae & 7 \\
\hline Pandalidae & 2 \\
\hline Panopeidae & 4 \\
\hline Penaeidae & 3 \\
\hline Pilumnidae & 2 \\
\hline Pinnotheridae & 1 \\
\hline Porcellanidae & 14 \\
\hline Portunidae & 5 \\
\hline Processidae & 1 \\
\hline Pseudorhombilidae & 1 \\
\hline Sesarmidae & 3 \\
\hline Sicyonidae & 2 \\
\hline Solenoceridae & 2 \\
\hline Upogebiidae & 3 \\
\hline Xanthidae & 6 \\
\hline \multicolumn{2}{|c|}{ STOMATOPODA } \\
\hline Gonodactylidae & 1 \\
\hline Squillidae & 3 \\
\hline TOTAL & 106 \\
\hline
\end{tabular}




\section{TABLE 2}

Species of crustaceans most frequently listed for the 19 stations, and stations where these were found; $\mathrm{B}$. Total number of species reported at each station.

\begin{tabular}{|c|c|c|}
\hline A. Species & Total & Station codes \\
\hline Neogonodactylus zacae & 4 & $6-8-10-12$ \\
\hline Alpheus rostratus & 3 & $6-8-12$ \\
\hline Clibanarius lineatus & 3 & $3-7-9$ \\
\hline Goniopsis pulchra & 3 & $3-7-9$ \\
\hline Hemus finneganae & 3 & $6-10-12$ \\
\hline Munida gracilipes & 3 & $1-G-I$ \\
\hline Palaemonella holmesi & 3 & $6-8-10$ \\
\hline Playpodiella rotundata & 3 & $6-8-12$ \\
\hline Pomatogebia rugosa & 3 & $6-8-12$ \\
\hline Processa peruviana & 3 & $8-12-1$ \\
\hline B. Station & $\begin{array}{l}1(1 \\
7(1 \\
(2), \\
\text { G ( }\end{array}$ & $\begin{array}{l}3(14), 4(3), 6(9), \\
9(15), 10(16), 11 \\
3(1), 14(2), F(10), \\
(6), L(1) M(3)\end{array}$ \\
\hline
\end{tabular}

\section{DISCUSSION}

Because almost all the specimens of macrocrustaceans from Golfo Dulce were collected during occasional visits, there is yet to be an intensive sampling effort covering most of the diverse habitats around its shores and in deep waters. The specimens came from a wide variety of habitats. The northern shore is characterized by decaying coral reefs and steep walls while the southern shore has mostly sandy beaches and mangrove swamps. The eastern shore has sandy beaches and a more extense shallow sandy platform. The only survey of deep waters was performed by the R.V. Victor Hensen expedition and it was restricted to a few stations.

Although sampling effort in Golfo Dulce has been relatively important, there is still much additional sampling needed to reach a reliable picture of the invertebrate biodiversity of the embayment. Of relevance are future collections in the area of Golfito Bay where only one entry is reported in the UCR Museum catalogue despite the relatively easy access to many of the environments around this shallow bay. Other relatively unexplored regions are intertidal and subtidal areas near Rincon Bay, the tip of the Osa peninsula, and the sandy beaches and shallow bottoms on the eastern shore. The fact that 77 species were found at only one station each supports the idea that each type of substrate appears to host a characteristic assemblage of species and emphasize the need to explore other sites characterized by different types of substrates.

According to Morales-Ramírez (2011), Golfo Dulce contains 21,5\% of the marine biodiversity of the Pacific coast of Costa Rica in an area of about $750 \mathrm{~km}^{2}$. He also reported a total of 1022 species of different animal groups (invertebrates and vertebrates) for Golfo Dulce. This total represents nearly 1,36 species per $\mathrm{km}^{2}$, a figure considered by Morales-Ramírez (2011) to be nearly double than that of the larger $\left(1990 \mathrm{~km}^{2}\right)$ and shallower Gulf of Nicoya estuary, where a more intensive sampling effort has been conducted (Vargas, 2016; Vargas-Zamora, Vargas-Castillo, \& Sibaja-Cordero, 2019)

Despite of some local pollution problems (Spongberg \& Davis, 1998; Spongberg, 2004; García, Acuña-González, Vargas-Zamora, \& García-Céspedes, 2006; Spongberg et al., 2011), the biodiversity of Golfo Dulce appears to indicate a relatively healthy ecosystem (Morales-Ramírez et al., 2015). In this context updated information on the biodiversity of the estuary may contribute to a 
better management of its resources and attract interested visitors. In addition, updated lists are a first step to evaluate structural community changes due to local, regional or global stressors such as coastal development, pollution, and climate change. This list of selected groups of macrocrustaceans presented herein is a step towards these goals.

\section{ACKNOWLEDGMENTS}

We thank two anonymous reviewers for comments on an earlier draft, and Sergio Aguilar for preparing Fig. 1. This is a contribution of Zoology Museum, Biology School, University of Costa Rica

\section{REFERENCIAS}

Castro, M., \& Vargas, R. (1996) Annotated list of species of marine crustaceans (Decapoda and Stomatopoda) from Golfo Dulce, Costa Rica. Revista de Biología Tropical, 44 (Supplement 3), S87 - S95.

Cortés, J. (1990). The coral reefs of Golfo Dulce, Costa Rica: distribution and community structure. Atoll Research Bulletin, 344, 1 - 37. DOI: 10.5479/si.00775630.344.1

Córdoba, R., \& Vargas, J. A. (1996). Nutrient profiles at a 200 m deep station in Golfo Dulce, Costa Rica. Revista de Biología Tropical, 44 (Supplement 3), S233 - S236.

Dalsgaard, T., Canfield, D. E., Peterson, J., Thamdrup, B., \& Acuña-González, J. (2003). N2 production by the anammox reaction in the anoxic water column of Golfo Dulce, Costa Rica. Nature, 422, 606 - 608. DOI: $10.1038 /$ nature01526

García, V., Acuña González, J., Vargas Zamora, J. A., \& García Céspedes, J. (2006). Calidad bacteriológica y desechos sólidos en cinco ambientes costeros de Costa Rica. Revista de Biología Tropical, 54 (Supplement 1), S35 - S48.

Hebbeln, D., Beese, D., \& Cortés, J. (1996). Morphology and sediment structures in Golfo Dulce, Costa Rica. Revista de Biología Tropical, 44 (Supplement 3), S1 - S10

Jesse, S. (1996). Demersal crustacean assemblages along the Pacific coast of Costa Rica: a quantitative and multivariate assessment based on the Victor Hensen Costa Rica Expedition (1993 / 1994). Revista de Biología Tropical, 44 (Supplement 3), S115 - S134.

León-Morales, R., \& Vargas, J. A. (1998). Macroinfauna of a tropical fjord-like embayment, Golfo Dulce, Costa Rica. Revista de Biología Tropical, 46 (Supplement 6), S81 - S90.

Morales-Ramírez, A. (2011). La diversidad marina del Golfo Dulce, Pacífico sur de Costa Rica: amenazas a su conservación. Biocenosis, 24, 9 - 20.

Morales-Ramírez, Á., Acuña-González, J., Lizano, O., Alfaro, E., \& Gómez, E. (2015). Rasgos oceanográficos en el Golfo Dulce, Pacífico de Costa Rica: una revisión para la toma de decisiones en conservación marina. Revista de Biología Tropical, 63 (Supplement 1), S131 - S160.

Samper-Villarreal, J., \& Silva-Benavides, A. M. (2015). Complejidad estructural de los manglares de Playa Blanca, Escondido y Rincón de Osa, Golfo Dulce, Costa Rica. Revista de Biología Tropical, 63 (Supplement 1), S199 - S208.

Svendsen, H., Rosland, R., Myking, S., Vargas, J. A., Lizano, O. G., \& Alfaro, E. J. (2006). A physical-oceanographic study of Golfo Dulce, Costa Rica. Revista de Biología Tropical, 54 (Supplement 1), S147 - S170.

Spongberg, A. I., \& Davis, P. (1998). Organochlorinated pesticide contaminants in Golfo Dulce, Costa Rica. Revista de Biología Tropical, 46 (Supplement 6), S111 - S124. 
Spongberg, A. I. (2004). PCB contamination in marine sediments from Golfo Dulce, Pacific Costa Rica. Revista de Biología Tropical, 52 (Supplement 2), S23 - S32.

Spongberg, A. L., Witter, J. D., Acuña, J., Vargas, J. A., Murillo, M., Umaña, G., Gómez, E., \& Pérez, G. (2011). Reconnaissance of selected Pharmaceutical and Personal Care Product compounds in Costa Rican surface waters. Water Research, 45, 6709 - 6717. DOI: 10.1016/j.watres.2011.10.004

Vargas, R., Jesse, S., \& Castro, M. (1996). Checklist of crustaceans (Decapoda and Stomatopoda), collected during the Victor Hensen Costa Rica Expedition (1993 / 1994). Revista de Biología Tropical, 44 (Supplement 3), S97 - S102.

Vargas, J. A. (2016). The Gulf of Nicoya estuarine ecosystem. In. M. Kaapelle (Ed.). Ecosystems of Costa Rica Chicago (pp. 106-124). Chicago, USA: University of Chicago Press.

Vargas-Zamora J. A., Vargas-Castillo, R., \& Sibaja Cordero, J. A. (2019). Crustáceos (Decapoda y Stomatopoda) del R.V. Skimmer y R.V. Victor Hensen en el Golfo de Nicoya, Pacifico. Costa Rica. Revista de Biología Tropical, 67, 286 305. DOI: 10.15517/rbt.v67i1.34729

Wolff, M., Hartmann, H. J., \& Koch, V. (1996). A pilot trophic model for Golfo Dulce: a fjord-like embayment, Costa Rica. Revista de Biología Tropical, 44 (Supplement 3), S215 - S231. 


\section{APPENDIX 1}

List of species of Decapoda and Stomatopoda collected in Golfo Dulce and deposited in the collection of the Museum of Zoology (MZUCR), University of Costa Rica: $E=$ endosymbiont in a clam, I = intertidal, $C R=$ coral rock, GD = Golfo Dulce, $S$ = sediment, $\mathrm{SC}=$ soft coral, $\mathrm{M}=$ mangrove, $\mathrm{PT}=$ polychaete tubes, $\mathrm{R}=$ rocks, $\mathrm{WR}=$ worm reef. No data $=$ ?

\begin{tabular}{|c|c|c|}
\hline & Species & $\begin{array}{l}\text { Catalog code, station, depth, substrate, } \\
\text { year of collection }\end{array}$ \\
\hline \multicolumn{3}{|c|}{ DECAPODA } \\
\hline 01. & Acantholobulus mirafloresensis Abele \& Kim, 1989 & 1455-01 / 3 / 0 m / ? / 1990 \\
\hline 02. & Achelous asper (A. Milne-Edwards, 1861) & $\begin{array}{l}\text { 2104-06 / F / } 70 \text { m / S / } 1994 \\
\text { 207-01 / } 14 \text { / } 18 \text { m / s / } 1969\end{array}$ \\
\hline 03. & Achelous iridescens (Rathbun, 1894) & $2005-06$ / I / 160-200 m / S /1993 \\
\hline 04. & Ala cornuta (Stimpson, 1860) & $\begin{array}{l}\text { 3162-17 / } 12 \text { / 1-2 m / CR / } 2013 \\
3483-01 \text { / } 12 \text { / ? / CR / } 2013\end{array}$ \\
\hline 05. & Alpheus bellimanus Lockington, 1877 & $3204-01$ / 10 / 27 m / CR / 2012 \\
\hline 06. & Alpheus floridanus Kingsley, 1878 & 2006-02 / G / 200 m / S /1994 \\
\hline 07. & Alpheus hebes W. Kim \& Abele, 1988 & $2929-02$ / 10 / 0 m / I / 2012 \\
\hline 08. & Alpheus pacificus Dana, 1852 & $2929-03$ / 10 / 0 m / I / 2012 \\
\hline 09. & Alpheus panamensis Kingsley, 1878 & 3162-03 / 12 / 1-2 m / CR / 2013 \\
\hline 10. & Alpheus rostratus W. Kim \& Abele, 1988 & $\begin{array}{l}\text { 3405-06 / } 6 \text { / 8-10 m / CR / } 2013 \\
3185-08 \text { / } 8 \text { / 1-2 m / CR / } 2013 \\
3162-04 \text { / } 12 \text { / 1-2 m / CR / } 2013\end{array}$ \\
\hline 11. & Aratus pacificus Thiercelin \& Schubart, 2014 & 499-01 / 7 / 0 m / M / 1969 \\
\hline 12. & Calcinus obscurus Stimpson, 1859 & $\begin{array}{l}\text { 1370-06 / } 3 \text { / } 0 \text { m / I, R, M /1990 } \\
1368-06 \text { / } 9 \text { / } 0 \text { m / M / } 1990\end{array}$ \\
\hline 13. & Callianidea mariamartae Hernaez \& Vargas, 2013 & $\begin{array}{l}\text { 2928-01-02 / } 10 \text { / } 0 \text { m / I, R / } 2012 \\
2929-01 \text { / } 10 \text { / } 0 \text { m / I, R / } 2012 \\
2957-01 \text { / } 10 \text { / } 0 \text { m / I, R / } 2012\end{array}$ \\
\hline 14. & Callinectes arcuatus Ordway, 1863 & $\begin{array}{l}\text { 2104-05 / F / } 70 \text { m / S / } 1994 \\
502-01 \text { / } 7 \text { / ? / ? / } 1969\end{array}$ \\
\hline 15. & Cancer johngarthi Carvacho, 1984 & $2005-00$ / I /160-200 m / s /1993 \\
\hline
\end{tabular}




\begin{tabular}{|c|c|c|}
\hline 16. & Cardisoma crassum Smith, 1870 & $\begin{array}{l}\text { 434-01 / } 7 \text { / } 0 \text { m / ? / } 1969 \\
\text { 515-01 / } 7 \text { / } 0 \text { m / ? / } 1969 \\
1365-02 \text { / } 7 \text { / } 0 \text { m / M / } 1990\end{array}$ \\
\hline 17. & Chasmocarcinus latipes Rathbun, 1898 & $\begin{array}{l}\text { 1995-04 / F / } 70 \text { m / S /1993 } \\
\text { 2006-03 / G / } 200 \text { m / } 1994\end{array}$ \\
\hline 18. & Clibanarius albidigitus Nobili, 1901 & 1370-05 / 3 / 0 m / I, R, M /1990 \\
\hline 19. & Clibanarius lineatus (H. Milne Edwards, 1848) & $\begin{array}{l}\text { 870-01 / } 7 \text { / } 0 \text { m / ? / } 1969 \\
1370-04 \text { / } 3 \text { / } 0 \text { m / I, R, M /1990 } \\
1368-07 \text { / } 9 \text { / } 0 \text { m / M / } 1990\end{array}$ \\
\hline 20. & Cronius ruber Lamarck, 1818 & 2846-02 / 6 / ? / ? / 2011 \\
\hline 21. & Cyrtoplax schmitti Rathbun, 1935 & $\begin{array}{l}\text { 1446-01 / } 9 \text { / } 0 \text { m / ? / } 1990 \\
1447-01 \text { / } 9 \text { / } 0 \text { m / ? / } 1990 \\
1454-01 \text { / } 11 \text { / } 0 \text { m / M / } 1990 \\
1456-02 \text { / } 11 \text { / } 0 \text { m / ? / } 1990\end{array}$ \\
\hline 22. & Dardanus nudus Ayón-Parente \& Hendrickx, 2009 & 1995-05 / F / 70 m / S /1993 \\
\hline 23. & Dardanus sinistripes (Stimpson, 1858) & 2007-03 / H / 15-20 m / S / 1993 \\
\hline 24. & Emerita rathbunae Schmitt, 1935 & 2794-01 / GD / 0 m / ? / 1995 \\
\hline 25. & Epixanthus tenuidactylus (Lockington, 1877) & 1370-02 / 3 / 0 m / I, R, M /1990 \\
\hline 26. & Eurypanopeus canalensis Abele \& Kim, 1989 & $\begin{array}{l}1453-02 \text { / } 9 \text { / } 0 \text { m / ? / } 1990 \\
1466-04 \text { / } 9 \text { / } 0 \text { m / ? / } 1990\end{array}$ \\
\hline 27. & Eurypanopeus transversus (Stimpson, 1860) & $\begin{array}{l}448-01 \text { / } 7 \text { / } 0 \text { m / M / } 1969 \\
1452-02 \text { / } 3 \text { / } 0 \text { m / ? / } 1990\end{array}$ \\
\hline 28. & Eurytium tristani Rathbun, 1906 & $\begin{array}{l}\text { 501-01 / } 7 \text { / } 0 \text { m / ? /1969 } \\
1369-02 \text { / } 7 \text { / } 0 \text { m / M / } 1990\end{array}$ \\
\hline 29. & Gnathophyllum panamense (Faxon, 1893) & $3162-06$ / 12 / 1-2 m / CR / 2013 \\
\hline 30. & Goniopsis pulchra Lockington, 1877 & $\begin{array}{l}\text { 498-01 / } 7 \text { / } 0 \text { m / M / } 1969 \\
1362-05 \text { / } 7 \text { / } 0 \text { m / M / } 1990 \\
1366-02 \text { / } 9 \text { / } 0 \text { m / M / } 1990\end{array}$ \\
\hline
\end{tabular}




\begin{tabular}{|c|c|c|}
\hline & & $\begin{array}{l}\text { 1368-04 / } 9 \text { / } 0 \text { m / M / } 1990 \\
1452-01 \text { / } 3 \text { / } 0 \text { m / ? / } 1990\end{array}$ \\
\hline 31. & Hemus finneganae Garth, 1958 & $\begin{array}{l}\text { 3077-03 / } 10 \text { / } 27 \text { m / R / } 2012 \\
3162-18 \text { / } 12 \text { / 1-2 m / CR / } 2013 \\
3405-08 \text { / } 6 \text { / 8-10 m / CR / } 2013\end{array}$ \\
\hline 32. & Hepatus kossmanni Neumann, 1878 & $266-01$ / 14 / 18 m / S / 1969 \\
\hline 33. & Heterocarpus vicarius Faxon, 1893 & $2005-02$ / I / 160-200 m / S /1993 \\
\hline 34. & Hirsutodynomene ursula (Stimpson, 1860) & 1995-02 / F / 70 m / S / 1993 \\
\hline 35 & Leptuca oerstedi (Ratbun, 1904) & $1362-04 / 7$ / 0 m /1990 \\
\hline 36. & Leptuca terpsichores (Crane, 1941) & $1463-01 / 3 / 0$ m / S / 1990 \\
\hline 37. & Leucosilia jurinii (de Saussure, 1853) & $500-01 / 7$ / 0 m / M /1969 \\
\hline 38. & Lysmata californica (Stimpson, 1856) & 2007-02 / H / 15-20 m / S /1993 \\
\hline 39. & Megalobrachium erosum (Glassell, 1936) & $3162-14$ / 12 / 1-2 m / CR / 2013 \\
\hline 40. & Megalobrachium pacificum (Gore \& Abele, 1974) & $\begin{array}{l}\text { 1966-01 / } 3 \text { / ? / ? / } 1990 \\
\text { 2402-01 /10 / ? / WR / } 1997\end{array}$ \\
\hline 41. & Microcassiope xantusii Stimpson, 1871 & 3405-09 / 6 / 8-10 m / CR / 2013 \\
\hline 42. & Munida gracilipes Faxon, 1893 & $\begin{array}{l}\text { 2005-03 / I / 160-200 m / S /1993 } \\
\text { 2006-01 / G / } 200 \text { m / S / } 1994 \\
3578-01 \text { / } 01 \text { / ? / S / } 2004\end{array}$ \\
\hline 43. & Ocypode gaudichaudii H. Milne Edwards \& Lucas,1843 & $1370-03 / 3 / 0$ m / I, R, M /1990 \\
\hline 44. & Orthochela pumila Glassell, 1936 & $3312-01$ / 2 / 11,3 m / SC / 2013 \\
\hline 45. & Ozius perlatus Stimpson, 1860 & 2402-03 /10 / ? / WR / 1997 \\
\hline 46. & Ozius verreauxii Saussure, 1853 & $\begin{array}{l}\text { 1368-05 / } 9 \text { / } 0 \text { m / M / } 1990 \\
\text { 2131-01 / } 9 \text { / } 0 \text { m / ? / } 1990 \\
1367-01 \text { / } 10 \text { / } 0 \text { m / R / } 1990\end{array}$ \\
\hline 47. & Pachygrapsus transversus (Gibbes, 1850) & $\begin{array}{l}\text { 1368-02 / } 9 \text { / } 0 \text { m / M / } 1990 \\
1362-06 \text { / } 7 \text { / } 0 \text { m / M /1990 }\end{array}$ \\
\hline 48. & Paguristes holmesi Glassell, 1937 & $1970-01$ / G / 200 m / S /1994 \\
\hline
\end{tabular}




\begin{tabular}{|c|c|c|}
\hline 49. & Pagurus virgulatus (Haig \& Harvey, 1991) & 3185-11 / 8 / 1-2 m / CR / 2013 \\
\hline 50. & Palaemon gracilis (Smith, 1871) & $1145-01 / 11$ / 0.5 m / S / 1979 \\
\hline 51. & Palaemonella holmesi (Nobili, 1907) & $\begin{array}{l}\text { 3077-06 / } 10 \text { / } 27 \text { m / R / } 2012 \\
2846-01 \text { / } 6 \text { / ? / ? / } 2011 \\
3405-07 \text { / } 6 \text { / 8-10 m / CR / } 2013 \\
3185-02 \text { / 8 / 1-2 m / CR / } 2013\end{array}$ \\
\hline 52. & Panopeus purpureus Lockington, 1877 & $\begin{array}{l}\text { 503-01 / } 7 \text { / } 0 \text { m / ? / } 1969 \\
1368-03 \text { / } 9 \text { / } 0 \text { m / M / } 1990 \\
1369-01 \text { / } 7 \text { / } 0 \text { m / M / } 1990\end{array}$ \\
\hline 53. & Pantomus affinis Chace, 1937 & $\begin{array}{l}2005-01 \text { / I / } 200 \text { m / S /1993 } \\
2009-01 \text { / I / 160-200 m / s /1993 }\end{array}$ \\
\hline 54. & Penaeus brevirostris Kingsley, 1878 & 2008-01 / M / 80-110 m / S /1993 \\
\hline 55. & Penaeus californiensis Holmes, 1900 & 2008-01 / M / 110 m / S /1993 \\
\hline 56. & Periclimenaeus spinosus Holthuis, 1951 & 3405-05 / 6 / 8-10 m / CR / 2013 \\
\hline 57. & Periclimenes infraspinis (Rathbun, 1902) & $\begin{array}{l}\text { 2243-1-2-3 / } 10 \text { / } 9.5 \text { m / SC / } 1997 \\
3187-03 \text { / } 10 \text { / ? / SC /1997 }\end{array}$ \\
\hline 58. & Persephona subovata (Rathbun, 1894) & 262-01 / GD / 90 m / ? /1969 \\
\hline 59. & Petramithrax pygmaeus Bell, 1835 & $\begin{array}{l}\text { 3162-01 / } 12 \text { / 1-2 m / CR / } 2013 \\
3478-01 \text { /12 / ? / CR / } 2013\end{array}$ \\
\hline 60. & Petrolisthes agassizii Faxon, 1895 & 3162-13 / 12 / 1-2 m / CR / 2013 \\
\hline 61. & Petrolisthes armatus (Gibbes, 1850) & $\begin{array}{l}1465-01 \text { / } 9 \text { / } 0 \text { m / ? / } 1990 \\
1466-05 \text { / } 9 \text { / } 0 \text { m / ? / } 1990 \\
1817-01 \text { / } 3 \text { / } 0 \text { m / s / } 1990 \\
2194-02 \text { / } 3 \text { / } 0 \text { m / ? / } 1997\end{array}$ \\
\hline 62. & Petrolisthes glasselli Haig, 1957 & $3162-16$ / 12 / 1-2 m / CR / 2013 \\
\hline 63. & Petrolisthes robsonae Glassell, 1945 & 2194-03 / 3 / 0 m / ? / 1997 \\
\hline 64. & Petrolisthes tonsorius Haig, 1960 & 1466-01 / 9 / 0 m / ? / 1990 \\
\hline 65. & Petrolisthes tridentatus Stimpson, 1859 & 1466-02 / 9 / 0 m / ? / 1990 \\
\hline
\end{tabular}




\begin{tabular}{|c|c|c|}
\hline 66. & Petrolisthes zacae Haig, 1968 & $\begin{array}{l}\text { 2227-01 / } 7 \text { / } 0 \text { m / M / } 1990 \\
2232-01 \text { / } 7 \text { / } 0 \text { m / M / } 1990\end{array}$ \\
\hline 67. & Pilumnus limosus Smith, 1869 & $\begin{array}{l}\text { 3162-21 / } 12 \text { / 1-2 m / CR / } 2013 \\
\text { 3185-05 / } 8 \text { / 1-2 m / CR / } 2013\end{array}$ \\
\hline 68. & Pilumnus townsendi Rathbun, 1923 & $\begin{array}{l}\text { 3077-02 / } 10 \text { / } 27 \text { m / R / } 2012 \\
3108-01 \text { / } 4 \text { / 12-22 m / CR / } 2012\end{array}$ \\
\hline 69. & Pinnixa longipes (Lockington, 1876) & 3413-01 / 10 / 10 m / PT / 2014 \\
\hline 70. & Pisidia magdalenensis (Glassell, 1936) & $3162-15$ / 12 / 1-2 m / CR / 2013 \\
\hline 71. & Pitho quinquedentata Bell, 1835 & 3162-11 / 12 / 1-2 m / CR / 2013 \\
\hline 72. & Platyactaea dovii (Stimpson, 1871) & $3162-08$ / 12 / 1-2 m / CR / 2013 \\
\hline 73. & Platypodiella rotundata (Stimpson, 1860) & $\begin{array}{l}\text { 3405-03 / } 6 \text { / 8-10 m / CR / } 2013 \\
3162-09 \text { / } 12 \text { / 1-2 m / CR / } 2013 \\
3185-06 \text { / } 8 \text { / 1-2 m / CR / } 2013\end{array}$ \\
\hline 74. & Pleuroncodes monodon (A. Milne Edwards, 1837) & $2005-07$ / I / 160-200 m / S /1993 \\
\hline 75. & Polyonyx nitidus Lockington, 1878 & 3108-05 / 4 / 12-22 m / CR / 2012 \\
\hline 76. & Polyonyx quadriungulatus Chace, 1956 & 3413-02 / 10 / 10 m / PT/ 2014 \\
\hline 77. & Pomatogebia rugosa (Lockington, 1878) & $\begin{array}{l}\text { 2200-01 / } 6 \text { / ? / ? / } 1996 \\
3162-23 \text { / } 12 \text { / 1-2 m / CR / } 2013 \\
3405-04 \text { / } 6 \text { / 8-10 m / CR / } 2013 \\
3185-03 \text { / } 8 \text { / 1-2 m / CR / } 2013\end{array}$ \\
\hline 78. & Pontonia margarita Verrill, 1869 & $\begin{array}{l}\text { 3186-01 / } 8 \text { / 1-2/ E / } 2013 \\
3188-01 \text { / } 8 \text { / 1-2 / E / } 2013\end{array}$ \\
\hline 79. & Portunus xantusii (Faxon, 1893) & 2104-02 / F / 70 m / S /1994 \\
\hline 80. & Processa peruviana Wicksten, 1983 & $\begin{array}{l}\text { 2005-06 / I / 160-200 m / } 1993 \\
\text { 3162-05 / } 12 \text { / 1-2 m / CR / } 2013 \\
\text { 3185-04 / } 8 \text { / 1-2 m / CR / } 2013\end{array}$ \\
\hline 81. & Pseudoveleronia laevifrons (Holthuis, 1951) & 3434-03 / 2 / 11. m / SC / 2013 \\
\hline 82. & Rimapenaeus pacificus Burkenroad, 1934 & 2008-02 / M / 80-110 m / 1993 \\
\hline
\end{tabular}




\begin{tabular}{|c|c|c|}
\hline 83. & Sesarma rhizophorae Rathbun, 1906 & $\begin{array}{l}\text { 1368-01 / } 9 \text { / } 0 \text { m / M / } 1990 \\
1366-03 \text { / } 7 \text { / } 0 \text { m / M / } 1990 \\
1365-06 \text { / } 7 \text { / } 0 \text { m / M / } 1990\end{array}$ \\
\hline 84. & Sesarma rubinofforum Abele, 1973 & 1365-08 / 7 / 0 m / M / 1990 \\
\hline 85. & Sesarma sulcatum Smith, 1870 & 1365-01 / 7 / 0 m / M / 1990 \\
\hline 86. & Sicyonia disedwardsi (Burkenroad, 1934) & $1995-01$ / F / 70 m / S /1993 \\
\hline 87. & Sicyonia picta Faxon, 1893 & $2005-04$ / I / 160-200 m / S /1993 \\
\hline 88. & Solenocera agassizii (Faxon, 1893) & $\begin{array}{l}\text { 2006-04 / G / } 200 \text { m / S /1994 } \\
2007-01 \text { / H / 15-20 m / S /1994 }\end{array}$ \\
\hline 89. & Solenocera mutator Burkenroad, 1934 & 2105-01 / L / 200 m / S /1993 \\
\hline 90. & Teleophrys cristulipes Stimpson, 1860 & $\begin{array}{l}\text { 3162-22 / } 12 \text { / 1-2 m / CR / } 2013 \\
3478-02 \text { /12 / ? / CR / } 2013\end{array}$ \\
\hline 91. & Tomopagurus merimaculosus (Glassell, 1937) & 2104-03 / F /70 m / S /1994 \\
\hline 92. & Tomopagurus purpuratus (Benedict, 1892) & $\begin{array}{l}\text { 1995-03 / F / } 70 \text { m / S / } 1993 \\
\text { 2104-04 / F / } 70 \text { m / s / } 994\end{array}$ \\
\hline 93. & Trachycaris restricta (A. Milne-Edwards, 1878) & 3108-03 / 4 / 12-22 m / CR / 2012 \\
\hline 94. & Uca heteropleura (Smith, 1870) & $1445-01$ / 9 / 0 m / ? / 1990 \\
\hline 95. & Uca princeps (Smith, 1870) & $1362-03 / 7$ / 0 m / s / 1990 \\
\hline 96. & Uca stylifera (H. Milne Edwards, 1852) & 1364-01 / 9 / 0 m / M / 1990 \\
\hline 97. & Ucides occidentalis (Ortmann, 1897) & $\begin{array}{l}\text { 413-01 / } 7 \text { / } 0 \text { m / ? / } 1969 \\
\text { 1399-01 / } 7 \text { / } 0 \text { m / M / } 1990\end{array}$ \\
\hline \multirow[t]{2}{*}{98.} & Uhlias ellipticus Stimpson, 1871 & 3162-10 / 12 / 1-2 m / CR / 2013 \\
\hline & & $3077-07$ / 10 / 27 m / R / 2012 \\
\hline 99. & Upogebia longipollex (Street, 1851) & $\begin{array}{l}\text { 3112-01 / } 10 \text { / } 0 \text { m / I / } 2012 \\
\text { 2402-02 / } 10 \text { / ? / WR / } 1997 \\
3210-01 \text { / } 10 \text { / } 0 \text { m / I / } 2012\end{array}$ \\
\hline 100. & Upogebia spinigera (Smith, 1871) & $\begin{array}{l}\text { 1727-01 / } 9 \text { / } 0 \text { m / ? / } 1990 \\
2194-01 \text { / } 3 \text { / } 0 \text { m / ? / } 1997\end{array}$ \\
\hline
\end{tabular}




\begin{tabular}{l|l|l}
\hline 101. & Williamstimpsonia stimpsoni (A. Milne-Edwards, 1879) & $3162-19$ / 12 / 1-2 m /CR / 2013 \\
\hline 102. & Xanthodius sternberghii (Stimpson,1859) & $1370-01$ / 3 / 0 m / I, R, M /1990 \\
\hline \multicolumn{2}{|c}{ STOMATOPODA } \\
\hline 103. & Neogonodacty/us zacae (Manning, 1972) & $3077-10$ / 10 / 27 m / R / 2012 \\
& & $3162-12$ / 12 / 1-2 m / CR / 2013 \\
& & $3185-07$ / 8 / 1-2 m / CR / 2013 \\
\hline 104. & Squilla biformis Biguelow, 1891 & $3405-11$ / 6 / 8-10 m / CR / 2013 \\
\hline 105. & Squilla hancocki Schmitt, 1940 & $2006-05$ / G / 200 m / S / 1994 \\
\hline 106. & Squilla panamensis Bigelow, 1891 & $195-02$ / GD / 101 m / ? / 1969 \\
\hline
\end{tabular}

